\title{
XIV. On the motion of bodies affected by friction. Communicated by Anthony Shepherd, D. D. F. R. S. Plumian Professor of Astronomy and Experimental Philosophy at Cambridge. Read November 25, 1784.
}

\section{Rev. Samuel Vince A. M.}

To cite this article: Rev. Samuel Vince A. M. (1803) XIV. On the motion of bodies affected by friction. Communicated by Anthony Shepherd, D. D. F. R. S. Plumian Professor of Astronomy and Experimental Philosophy at Cambridge. Read November 25, 1784., Philosophical Magazine Series 1, 17:66, 113-119, DOI: 10.1080/14786440308676379

To link to this article: http://dx.doi.org/10.1080/14786440308676379

\section{Published online: 18 May 2009.}

\section{Submit your article to this journal ๘}

Џ Article views: 2 
Mr. Cavallo has candidly acknowledged the difficulty of reconciling various properties of charged electrics with any received theory. Where, for instance, does the charge reside? Not in the coating, as may satisfactorily be shown : if in the glass, and the fluid can penetrate any the smallest portion, a glass might be made so thin that the fluid would freely pervade its substancc; but a glass ball $\frac{1}{60} \mathrm{t}^{\text {th }}$ of an inch thick will receive a powerful charge. The hypothesis still remains incumbered with numerous difficulties ; and it must be left to future investigation to determine whether it shall be wholly rejected, or whether subsequent discoveries may enable us to apply the foregoing principles with more certainty and success.

XIV. On the Motion of Bodies affected by Friction. By the Rev. Samuel Vince, A. M. of Cambridge. Communicated by Anthony Shepherd, D.D. F.R.S. Plumian Professor of Astronomy and Experimental Philosophy at Cambridge. Read November 25, 3784.

[Concluded from p. $5_{8 .}$ ]

PROPOSITION II.

LET the body be projected on an horizontal plane LM (fig. 3.) with a given velocity, to determine the space through which the body will move lefore it stops, or before its motion becomes uniform.

CASE I.-1. Suppose the body to have no rotatory motion when it begins to move; and let $a=$ the velocity of projection per second measured in feet, and let the retarding force of the friction of the body measured by the velocity of the body which it can destroy in one second of time, be determined by experiment and called $\mathrm{F}$, and let $x$ be the space through which the body would move by the time its motion was ali destroved when projected with the velocity $\mathrm{a}$, and retarded by a force $\mathrm{F}$; then, from the principles of uniformly retarded motion, $x=\frac{a^{2}}{2 \mathrm{~F}}$, and if $t=$ time of des scribing that space, we have $t=\frac{n}{\mathrm{~F}}$, and hence the space described in the first second of time $=\frac{2 a-F}{2}$. Now it is manifest that when the rotatory motion of the body about its axis is equal to its progressive motion, the point $a$ will be carried backwards by the former motion as much as it is

VoI. XVII. No. 66. 


\section{On the Motion of Bodies affected by Friction.}

carried forwards by the latter ; consequently the point of contact of the body with the plane will then have no moton in the direction of the plane, and hence the friction will at that instant cease, and tho body will continue to roll on uniformly witheut sliding with the velocity which it has at that point. Put therefore $z=$ the space described from the commencement of the motion till it becomes nniform, then the body being uniformly retarded, the spaces from the end of the motion vary as the squares of the velocities, hence $\frac{a^{2}}{2 \mathrm{~F}}: a^{2}(:: 1: 2 \mathrm{~F}):: \frac{a^{2}}{2 \mathrm{~F}}-z: a^{2}-2 \mathrm{~F} z=$ square of the progressive velocity when the motion becomes wiform; therefore the velocity destroyed by friction $=a-$ $\sqrt{u^{2}-2 F z}$; hcnce, as the velocity generated or destroyed in the same time is in proportion to the force, we have by Cor. 2. Prop. 1. $r s: r a:: a-\sqrt{a^{2}-2 \mathrm{Fz}}: \frac{r a}{r s} \times$ $a-\sqrt{a}-9+z$ the velocity of the circumference $e f g$ generased about the center, cousequently $\sqrt{a^{2}-2 \mathrm{~F} z}=\frac{r a}{r s} \times$ $\overline{a-\sqrt{d^{2}-2 \mathrm{~F} z}}$, and honce $\approx=\frac{\overline{r s^{2}+2 r s \times r a} \times a^{2}}{a s^{2} \times 2 \mathrm{~F}}$ the space which the body describes before the motion becomes uitiform.

s. If we substitute this value of $z$ into the expression for the rulecity, we shall have $a \times \frac{r a}{r s}$ for the velocity of the bcdy - when its motion becomes uniform; hence therefore it aiplears that the velceity of the body, when the friction cisce, will be the same whatever be the quantity of the friction. If the body be the circumferenes of a circle, it will alway lose half the relocity before its motion becomes mifom.

CASE If.-1. Let the body, besides having a progressive velocity in the direction LAI (fig. 3.) have also a rotatory motion about its center in the direction $g f e$, and let $v$ represent the initial velocity of any point of the circumference about the center, and suppose it first to be less than $a$; then friction being a uniformly retarding torce, no alteration of the velocity of the point of contact of the body upon the planc can affect the quantity of friction; hence the progressive relocity of the boay will be the same as before, and conscquent? the rotatory velocity generated by friction wilt also be the same; to which if we add the velocity about the 
center at the beginning of the motion, we shall have the whole rotatory motion; hence, therefore, $v+\frac{r a}{r s} \times$ $\overline{a-\sqrt{a^{2}-2} \bar{F} z}=\sqrt{a^{2}-2 \mathrm{~F}^{2}}$, consequently $z=$ $\frac{a^{2} \times a s^{2}-\sqrt{v \times r s+a \times r a^{2}}}{2 \mathrm{~F} \times a s^{2}}$ the space described before the motion becomes uniform.

2. If this value of $z$ be substituted into the expression for the velocity, we shall have $\frac{v \times r s+a \times r a}{a s}$ for the velocity when the friction ceases.

3. If $v=a$, then $z=0$, and hence the body will continue to move uniformly with the first velocity.

4. If $v$ be greater than $a$, then the rotatory motion of the point $a$ on the plane being greater than its progressive motion and in a contrary direction, the absolute motion of the point $a$ upon the plane will be in the direction ML, and consequently friction will now act in the direction LM in which the body moves, and therefore will accelerate the progressive and retard the rotatory motion; hence it appears that the progressive notion of a body may be ACCELERATED by friction. Now to determine the space described before the motion becomes uniform, we may observe that as the progressive motion of the body is now accelerated, the velocity after it has described any space $\approx$ will be $=\sqrt{a+2 \mathrm{~F} z}$, hence the velocity acquired $=\sqrt{a^{2}+2 \mathrm{~F} z}-a$, and con. sequently the rotatory velocity destroyed $\frac{r a}{r s} \times \sqrt{\sqrt{a^{2}+2 \mathrm{Fz}}-a}$, hence $v-\frac{r a}{r s} \times \overline{\sqrt{a^{2}+2 F z}-a}=\sqrt{a^{2}+g F z}$, therefore $\approx=\frac{\overline{r s \times v+r a \times a^{2}}-a^{2} \times a s^{2}}{2 \mathrm{~F} \times a s^{2}}$ the space required.

5. If $a=0$. or the body be placed upon the plane without any progressive velocity, then $z=\frac{r s^{2} \times v^{a}}{2 \overline{\mathrm{F}} \times a s^{2}}$.

CASE III.-1. Let the given rotatory motion be in the direction $g$ ef; then as the friction must in this case always act in the direction ML, it must continually tend to destroy both the progressive and rotatory motion. Now as the velocity destroyed in the same time is in proportion to the retarding force, and the force which retards the rotatory is to the furce which retards the progressive velooity by Cor. 2 . Prop. 1. as $r a: r s$, therefore if $v$ be to $a$ as $r a$ is to $r s$, $\mathrm{H} \nsubseteq$ then 


\section{On the Motion of Bodies affected by Friction.}

then the retarding forces being in proportion to the velocities, both morions will be destroyed together, and consequently the body, after describing a certain space, will rest; which space, being that described by the body uniformly retarded by the force $F$, will, from what was proved in Case I. be equal to $\frac{a^{2}}{2 \mathrm{~F}}$.

9. If $v$ bears a greater proportion to $a$ than $r a$ does to $r s$, it is manifest that the rotatory motion will not be all destroyed when the progressive is ; consequently the body, after it has described the space $\frac{a^{2}}{2 \mathrm{~F}}$, will return back in the direction MI, for the progressive motion being then destroved, and the rotatory motion still continuing in the direction $g$ e $f$, will cause the body to return with an accelcratite velocity until the friction ceases by the body's beginning to roll, after which it will move on uniformly. Now to determine the space described before this happens, we have $r s: r a:: a: \frac{r a \times a}{r s}$ the rotatory velocity destroyed when the progressive is all lost; henec $v-\frac{r a \times a}{r s}=$ $\frac{v \times r s-a \times r a}{r s}=$ the rotatory velocity at that time, which being substitnted for $v$ in the last article of Case II. gives $\frac{v+r-a \times r a}{2 \sqrt{1 \times a s^{2}}}$ for the space described before the motion becomes uniform.

3. If $"$ has a less proportion to $a$ than $r a$ has to $r s$, it is manifest that the rotatory motion will be destroyed betore the progersive; in which case a rotatory motion will be generated in a contrary direction until the two motions become equal, when the friction will instantly cease, and the body will then move on unifom!y. Now ra :rs::v: $\frac{v \times r s}{r a}$ the progressive velocity destroyed when the rotatory velocity ceases, hence $a-\frac{v \times r s}{r a}=\frac{a \times r a-v \times r s}{r a}=$ progressive velocity when it begins its rotatory motion in a contrary direction: substitute therefore this quantity for $a$ in the expression for $z$ in Case 1 . and we have $\frac{\frac{r s^{2}+2 r s \times r a}{a} \times \frac{a}{a+v \times r s}}{a s \times a r^{2} \times 2 F}$ for the space described after the rotatory motion ceases before the motion of the body becomes uniform. Now to determine the space described 
scribed before the rotatory motion was all destroyed, we have (as the space from the cnd of a uniformly retarded motion varies as the square of the velocity) $a^{2}: \frac{a^{2}}{2 F}:$ : $\frac{\overline{a \times r a-v^{\prime} \times r s}}{r a^{2}}: \frac{\overline{a \times r a-v \times r s}}{2 \mathrm{~F} \times r a^{2}}$ the space that could lave been described from the time that the rotatory velocity was destroyed, until the progressive motion would have been destroyed had the friction continued to act; hence $\frac{a^{2}}{2 \mathrm{~F}}-\frac{\overline{a \times r a-v \times r s}}{2 \mathrm{~F} \times r a^{2}}=\frac{2 a v \times r a \times r s-v^{2} \times r s^{2}}{2 \mathrm{~F} \times r a^{2}}=$ the space described when the rotatory motion was all destroyed, hence $\frac{\overline{r s^{2}+2 r s \times r a \times a \times a r-v \times s r}}{a s^{2} \times a r^{2} \times 2 \mathrm{~F}}+\frac{2 a v \times r a \times r s-v^{2} \times r s^{2}}{2 \mathrm{~F} \times r a^{2}}$ $=$ the whole space described by the body before its motion becomes uniform.

\section{DEFINITION.}

The Cen'res of Fricrion is that point in the lase of a body on which it revolves, into which if the whole surface of the base, and the mass of the bodiy were collected, "tiat" made to revolve about the center of the lase of the givm $7 ; i$, the angular velocity destroyed by its friction wontl ta to the angular velocity destroyed in the given boing lis its firction in the sume time.

\section{PROPOSITION III.}

To find the center of friction.

Let FGH (fig. 4.) be the base of a body revolving about its center $\mathrm{C}$, and suppose about $a, b, c$, \&ec. to be indefinitely small parts of the base, and let $A, B, C$, \&c. be the corresponding parts of the solid, or the prismatic parts having $a, b, c$, \&c. for their bases; and $P$ the center of friction. Now it is manifest that the decrement of the angular velocity must vary as the whole diminution of the momentum of rotation caused by the friction diectly, and as the whole momentum of rotation or effect of the inertia of all the particles of the solid inversely; the former being employed in diminishing the angular velocity, and the latter in opposing that diminution by the endearour of the particles to persevere in their motion. Hence, if the effect of the friction varies as the effect of the inertia, the decrements of the angular velocity in a given time will be equal. Now as the quantity of friction (as has been proved from experiments) does not depend on the velocity, the effect of If 3 


\section{On the Motion of Bodies affected by Friction.}

the friction of the elementary parts of the base $a, b, c$, \&e. will be as $a \times a \mathrm{C}, b \times b \mathrm{C}, c \times c \mathrm{C}$, \&c. also the effect of the inertia of the corresponding parts of the body will be as $\mathrm{A} \times a \mathrm{C}^{\circ}, \mathrm{B} \times \mathrm{l}^{\circ}, \mathrm{C} \times c \mathrm{C}^{\prime \prime}$, \&c. Now when the whole surface of the base and mass of the body are concentrated in $\mathrm{P}$, the effect of the friction will be as $\overline{a+b+c+}$

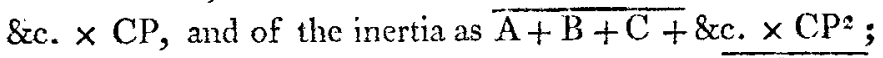
consequently $a \times a \mathrm{C}+b \times b \mathrm{C}+c \times c \mathrm{C}+\& \mathrm{xc} . \overline{a+b+c+}$ \&c. $\times \mathrm{CP}:: \mathrm{A} \times a \mathrm{C}^{2}+\mathrm{B} \times b \mathrm{C}^{2}+\mathrm{C} \times c^{2}+\& \mathrm{c}$. :

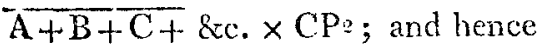
$\mathrm{CP}=\frac{\overline{\mathrm{A} \times a \mathrm{~B} \times} \mathrm{C}+\mathrm{C} \times \mathrm{C}^{2}+8 \mathrm{c} \times \overline{a+b+c+8 \mathrm{c}} .}{a \times a \mathrm{C}+b \times b \mathrm{C}+c \times c \mathrm{C}+\& \mathrm{c} \times \overline{\mathrm{A}+\mathrm{B}+\mathrm{C}+\& \mathrm{c}} .}$ $=$ (if $\mathrm{S}=$ the sum of the products of each particle into the square of its distance from the axis of motion, $\mathrm{T}=$ the sum of the products of each part of the base into its distance from the center, $s=$ the area of the base, $t=$ the solid content of the body) $\frac{\mathrm{S} \times s}{\mathrm{~T} \times t}$.

\section{PROPOSITION IV.}

Given the velocity with which a lody legins lo revolve about the center of its lase, to determine the numler of revolutions which the lody wivl make bofore all its motion be destroyed.

Let the friction, expressed by the velocity which it is able to destroy in the body if it were projected in a right line horizontally in one second, be determined by experiment, and called $\mathrm{F}$; and suppose the initial velocity of the center of friction $\mathrm{P}$ about $\mathrm{C}$ to be $a$. Then conceiving the whole surface of the base and mass of the body to be collected into the point $\mathrm{P}$, and (as has been proved in Prop. II.) $\frac{a^{2}}{2 \mathrm{~F}}$ will be the space which the body so concentrated will describe beture all its motion be destroyed; hence if we put $z=\mathrm{PC}, p=$ the circumference of a circle whose radius is unity, then will $p z=$ circumference described by the point $\mathrm{P}$; consequently $\frac{a^{?}}{2 b \approx \mathrm{F}}=$ the number of revolutions required.

Cor. If the sclid be a cylinder and $r$ be the radius of its base, then $z=\frac{3 r}{4}$, and therefore the number of revolutions $=\frac{2 \cdot a^{2}}{3 p r \mathrm{~F}}$. 


\section{PROPOSITION V.}

To find the nature of the curve descriled ly any point of a tody affected bif friction, when it descends down any inclined plane.

Let ef $g$ (fig. 5.) be the body, the points $\alpha, r, s$, as in Prop. I. and conceive $s t, r n$, to be two indefnitely small spaces described by the points $s$ and $r$ in the same time, and which therefore will represent the velocities of those points but from Prop. $\mathrm{I}$. the ratio of these velocities is expressed by $m \times \mathrm{CB}: a \times \mathrm{CA}$, bence $s t: r n:: m \times \mathrm{CB}: a \times$ CA. With the center $r$ let a circle $v w$ be deseribed touching the plane LM which is parallel to AC at the point $l$; and let the radius of this circle be such that, conceiving it to descend upon the plane LM along with the body alescending on $\mathrm{CA}$, the point $b$ may be at rest, or the circle may roll without sliding. To determine which radius, produce $r s$ to $x$, parallel to which draw $n d y$, and produce $n t$ to $\approx$; now it is manifest, that in order to answer the conditions above mentioned, the velocity of the point $x$ must be to the velocity of the point $r$ as $2: 1$, that is, $z x: y x$ $:: 2: 1$, hence $z y=y x=n r$. Now $z y: d t(:: n y:$ $n d):: r x: r s$; therefore $d t=\frac{r s}{r \cdot x} \times z y=\frac{r s}{r x} \times n r$, hence $t s\left(=t d+d s=t d+n r=\frac{r s}{r x} \times n r+n r\right)=\frac{r s+r x}{r x} \times n r$, consequently $\frac{r s+r x}{r x}: 1:: t s: n r::$ (from what is proved above) $m \times \mathrm{CB}: a \times \mathrm{CA}$; therefore $a \times \mathrm{CA} \times r s+a \times$ $\mathrm{CA} \times r^{r} x=m \times \mathrm{CB} \times r x$, hence $r x=\frac{a \times \mathrm{CA} \times r s}{m \times \mathrm{CB}-a \times \mathrm{CA}}$ the radius of the circle which rolling down the inclined plane LM, and carrying the body with it, will give the rue ratio of its progressive to its rotatory motion, and conscquently that point of the circle which coincides with any given point of the body will, as the circle revolves upon the line LM, describe the same curve as the corresponding point of the body; but as the nature of the curve described by any point of a circle revolving upen a straight line is already very well known, it seems unnecessary to give the investigation.

By a method of reasoning, not very different, may the nature of the curve, which is described by any point of a body moving upon an horizontal plane, and affected by friction, be determined. 